\title{
Cuidado de sí masculino o instrumentalización de los varones adultos jóvenes
}

\author{
Nora Eugenia Muñoz Franco \\ Universidad de Antioquia (Colômbia)
}

Cuidado de sí masculino o instrumentalización de los varones adultos jóvenes

Resumen: Este artículo tiene la finalidad de reconstruir la ruta que han transitado las políticas públicas colombianas en materia de cuidado de sí en la salud, y su relación con los varones jóvenes y las masculinidades, durante el período 1950-2000, identificando como un asunto central el hecho de que estas han sido diseñadas en torno al discurso del crecimiento económico y del posicionamiento de los varones como principal fuerza de trabajo del país. En tal sentido se instaura un dispositivo con dos mecanismos fundamentales para promover el cuidado de sí: mecanismos de salvaguarda de la capacidad productiva; y mecanismos de efectos periféricos. Se asume implícita en las políticas públicas la vinculación de los varones, en tanto estos tienen como epicentro los grupos poblacionales considerados vulnerables, tales como las mujeres y las niñas.

Palabras clave: Adultos jóvenes. Masculinidades. Cuidado de sí. Salud pública.

\section{Autocuidado masculino ou instrumentalização dos jovens adultos masculinos}

Resumo: O objetivo do artigo é reconstruir a rota que transitaram as políticas públicas colombianas no autocuidado em saúde e sua relação com os homens jovens e as masculinidades durante o período de 1950-2000, identificando como questão central o fato de que as mesmas foram concebidas em torno ao discurso do crescimento económico e do posicionamento dos homens como a principal força de trabalho no país. Neste sentido, se estabelece um dispositivo com dois mecanismos fundamentais para promover o autocuidado: mecanismos de salvaguarda da capacidade de produção e mecanismos de efeitos periféricos. Considera-se a vinculação dos homens como implícita nas políticas públicas, enquanto elas têm como epicentro os grupos populacionais considerados vulneráveis, como as mulheres e as meninas.

Palavras-chave: Jovens adultos. Masculinidades. Autocuidado. Saúde pública.

\section{Men Caring for Themselves: the instrumentalization of young adult males}

Abstract: The purpose of this article is to reconstruct the route taken by Colombian public policies in relation to care for one's own health, and its relation with young males and masculinities, from 1950 to 2000. It identifies as a central issue the fact that these policies have been designed around the discourse of economic growth and the positioning of the male as the main labor force in the country. In this sense a device was established with two fundamental mechanisms for promoting care for oneself: mechanisms to protect productive capacity; and mechanisms with peripheral effects. The connection with males is implicit in public policies, while they have as their main focus the population groups considered to be vulnerable, such as women and children.

Keywords: Adult youth. Masculinities. Care for oneself. Public health. 


\section{Presentación}

En el ámbito del Trabajo Social, es claro que uno de los escenarios privilegiados para la intervención profesional ha sido el área de la salud, sobre todo si se alude a los problemas y fenómenos asociados con los colectivos humanos. Se trata de la Salud pública como aquella en la cual se materializan las políticas públicas en salud. Por ello, a través de este artículo ${ }^{1}$ se generan aportes en los que se describen y se cuestionan las acciones y el dispositivo que desde el Estado se ha desarrollado en relación con el cuidado de si en la salud masculina, con la finalidad de analizar sus lógicas de funcionamiento interno, así como los mecanismos y estrategias que permiten su eficacia como discurso de saber/poder que circula entre los varones, sobre todo los adultos jóvenes, y en la sociedad en general. Política y económicamente, los procesos de desarrollo exógeno que ha vivido el país han limitado las posibilidades internas de organización de la vida nacional, supeditando los esfuerzos de este a diversas demandas de organismos internacionales y de naciones como los Estados Unidos, que han tenido la clara intención de ejercer el control y el poder sobre el territorio y sus recursos (LÓPEZ, 2011), asuntos que claramente delimitan y condicionan las formas de ejercer la profesión del Trabajo Social.

Este artículo tiene la finalidad de reconstruir la ruta que han transitado las políticas públicas colombianas en materia de cuidado de sí en la salud, y su relación con los varones jóvenes y las masculinidades, identificando como un asunto central el hecho de que estas han sido diseñadas en torno al discurso del crecimiento económico y del posicionamiento de los varones como principal fuerza de trabajo del país, es decir, se han ubicado en el escenario productivo (PELÁEZ; RODAS, 2002) en contraposición con las mujeres, quienes preponderantemente han sido instaladas en el ámbito de la reproducción y la vida doméstica. Tal planteamiento conlleva el reforzamiento, desde la institucionalidad, de los parámetros de género que dominan el orden social colombiano. Género y cuidado de sí se entrecruzan desde las políticas públicas para convertirse en un dispositivo de control y sometimiento de los jóvenes, tanto varones como mujeres, de acuerdo con requerimientos políticos y económicos que se conjugan a través de diferentes formas de institucionalización, se trata pues de una racionalidad económica en esencia. Pero, para identificar las formas en que opera la racionalidad a la que se alude en Colombia, este artículo parte de proponer dos elementos interdependientes que son fundamentales para hacer una lectura crítica de la configuración del cuidado de sí en la salud masculina, entre los años 1950 y 1990. Los elementos a los que se hace referencia son la política pública como proceso sociopolítico y el carácter político del cuidado de sí, porque ello lo convierte en objeto y fin de la política. En este sentido, la opción sería adoptar políticas públicas saludables, es decir, políticas públicas en todas las áreas y sectores preocupadas por el impacto que sus acciones pueden acarrear para la salud y la equidad entre hombres y mujeres. El Cuidar de sí y la reflexividad terminan siendo mecanismos para desdibujar los límites entre el adentro y el afuera, en otras palabras, entre el yo y el otro, y también para quebrar las fronteras mismas tanto del adentro como del afuera, las de la subjetividad y las del mundo. Ésta es, precisamente, la fuerza, la potencia, la condición ético-política del cuidado de sí (CUBIDES CIPAGAUTA, 2006).

Son entonces las relaciones con la sociedad y por ende con instituciones como el Estado, la familia y la escuela, entre otras, las que posibilitan la construcción de ciertas actitudes y prácticas crítico-reflexivas no solo individuales, sino colectivas, para el cuidado de la salud, hecho que le da a éste un carácter político. Se debiera buscar entonces "desarrollar la dimensión cultural de la política, lo cual implica construir la experiencia subjetiva del nosotros y potenciar nuestras capacidades para organizar las formas en que queremos convivir" hombres y mujeres. El reto por consiguiente, es "experimentar nuevas formas de política vinculadas a una ética agonal y a una política del arte de vivir" (CUBIDES CIPAGAUTA, 2006, p. 4). Es en este contexto que se encauzaron los destinos de Colombia en el sector salud durante la segunda mitad del siglo 20, incluidas las acciones y políticas que orientarían, implícita o explícitamente, las actitudes y prácticas del cuidado de la salud tanto en los varones como en las mujeres. Actitudes y prácticas estas desarrolladas de manera naturalizada bajo subterfugios y sutilezas que albergaron la perversa extensión de un poderío que controla, de manera discreta pero apabullante el espacio, los comportamientos, los cuerpos, la vida. El género en este contexto adquiere importancia capital en tanto debe partirse del reconocimiento de que éste continúa siendo un marcador que explica la inequidad social, máxime cuando en las políticas macroeconómicas por ejemplo, no es clara la inclusión de esta perspectiva, hecho que influencia y condiciona las decisiones, los recursos y las acciones en salud.

Se configura así un dispositivo de poder en los límites del territorio como asunto asociado a la intromisión en él por parte de organismos multilaterales como el Banco Mundial y el Banco Interamericano de Desarrollo principalmente, que, con una visión ajena a las realidades nacionales y bajo una racionalidad económica, introducen lineamientos que condicionan el diseño y la implementación de las políticas públicas. Tales lineamientos están desprovistos de una perspectiva que valore la importancia de involucrar el género desde la equidad y garantía de la participación proactiva de hombres y mujeres en los procesos sociopolíticos del país, bajo su condición de sujetos de derechos. 
Para el caso de los varones, el dispositivo planteado opera desde dos ángulos de operación: uno directo y explícito que denomino mecanismos de salvaguarda de la capacidad productiva, que, bajo la lógica del costobeneficio, conlleva la implementación de estrategias y tácticas asociadas con la prevención, la disuasión, la vigilancia, la protección y el control para garantizar la efectividad de los varones adultos jóvenes en los procesos de producción, minimizando los costos por incapacidad y enfermedad. Estos mecanismos contribuyen a entender por qué las políticas públicas que se interesan por los varones, hacen énfasis en el grupo etario de los adultos jóvenes, que cobijan el rango de edad de los 15 a los 44 años, por ser considerado el grupo en el que mayoritariamente se concentra la población económicamente activa (PEA) (FLÓREZ, 2000).

El segundo opera de manera indirecta y lo denomino mecanismos de efectos periféricos, porque se asume implícito en las políticas públicas en tanto estos tienen como epicentro los grupos poblacionales considerados vulnerables: las mujeres, los/las niñas, la tercera edad, entre otros, pero cuyos efectos pueden permear a los hombres. Las estrategias específicas desde este ángulo se asocian con el mantenimiento del cuerpo, con una serie de acciones intencionadas en relación con el régimen alimenticio, la regulación y/o prohibición de los consumos (cigarrillo, sustancias psicoactivas, alcohol) y la salud mental. Así mismo, se ubica en esta línea la restricción del erotismo y la sexualidad, con énfasis en el grupo de adultos jóvenes.

El dispositivo que se analiza en torno al cuidado de sí y sus mecanismos de aplicabilidad en la población adulta joven masculina, proveyó al Trabajo Social de herramientas que posibilitaron mayor tecnificación de las acciones profesionales en salud, en la medida en que mediante su desarrollo se aportaron nuevas estrategias de intervención preventiva, promocional y educativa en el ámbito de la salud pública en las cuales profesionales del área de las Ciencias Sociales, tales como el o la Trabajadora Social, fueron los llamados a coordinar su operativización.

Dado lo anterior, resulta relevante plantear las estrategias y tácticas que se crearon y se pusieron en marcha para el logro del control y la protección de la población masculina económicamente activa, mediante los discursos del cuidado de sí instituidos, lo que tácitamente direcciona la acción profesional a lo largo de las décadas en estudio y proyecta elementos de análisis del contexto socio-político y económico actual en el cual se desarrolla la intervención desde Trabajo Social.

\section{Enfoque Metodológico}

La investigación enfatizó en un análisis documental que privilegió los discursos de tres periódicos de circulación nacional en Colombia (El Espectador, El Tiempo, y El Colombiano) y documentos de organismos multilaterales del orden nacional e internacional, acerca de la salud pública en relación con el cuidado de sí masculina en el período 1950-2000. Con elementos de la genealogía foucaultiana se construyó el enfoque metodológico del estudio que alude al análisis de las formas de ejercicio del poder. Éste permitió indagar por la procedencia y la emergencia de las cosas buscando los rastros y huellas que dejan los procesos de los que son producto y que siguen teniendo incidencia en el presente. La genealogía, como relectura de la historia, se fundó para este trabajo en el análisis de materiales históricos (documentos, material audiovisual etc.) que se recuperaron del pasado. Mediante la genealogía se buscó romper las continuidades de la historia y hacer una crítica a las instituciones que rigen el presente como a la ciencia estatutaria, que establece el discurso hegemónico o aceptado como verdadero.

Como estrategia se utilizó el análisis del discurso para identificar las formas del ejercicio del poder que en él se desarrolla. A través de la genealogía se buscó, en el discurso, un campo de regularidad para distintas posiciones de subjetividad, permitiendo identificar la táctica utilizada. Se muestra, con base en este enfoque, la gama de mecanismos en torno al cuidado de sí masculino, propuestos por la salud pública instituida.

\section{Mecanismos de salvaguarda de la capacidad productiva}

Este mecanismo es el que mayores desarrollos ha tenido en el ámbito nacional en relación con el cuidado de la salud en la población masculina, por las implicaciones que los riesgos en el trabajo pueden tener para la economía del país. Así, a lo largo de la segunda mitad del siglo 20, se fue desarrollando una serie de normas, reglamentos, procedimientos y proposiciones científicas que contribuyeron a la consolidación de un poder soportado por el saber especializado de la seguridad industrial que transitó a lo que hoy se conoce como salud ocupacional. Estos desarrollos se debieron a los altos reportes de accidentalidad en los trabajadores.

Cobran fuerza entonces los discursos de la Seguridad Industrial, que buscan salvaguardar los escenarios de trabajo y, lógicamente, la capacidad productiva de los trabajadores. Estas medidas se instauran principal- 
mente en ámbitos laborales de predominio masculino, tales como los talleres, la industria metalúrgica y metalmecánica. El cuidado de sí se orienta hacia el control del riesgo y busca, mediante estrategias de prevención y capacitación, desarrollar habilidades en los trabajadores para el manejo de herramientas, metales (EL ESPECTADOR, 1960), mantenimiento de los lugares de trabajo, levantamiento de cargas (EL ESPECTADOR, 1971), medidas de protección para trabajar maderas, manejo de equipos (EL ESPECTADOR, 1973), la importancia del casco protector e inspecciones de seguridad con el objetivo de ejercer el control de posibles accidentes que alteren la calidad de la producción, entre muchas otras. En estos discursos priman dos elementos fundamentales: el primero, se asocia a las pérdidas de las empresas por concepto de lesiones entre sus trabajadores y daños a la maquinaria. El segundo, directamente relacionado con sostener la fuerza de trabajo, por considerarla fundamental para su productividad. Se adoptan prácticas de autocuidado que eliminen cualquier acto inseguro, teniendo en cuenta que tanto el empresario como los trabajadores, deben apuntarle al mismo objetivo. En este sentido, un acto inseguro se entiende como aquel que se ocasiona cuando una persona incumple las normas que han sido aceptadas como seguras, o aquellas que aún sin estar escritas son obvias para el sentido común: "hay muchas causas del acto inseguro, entre las más importantes: impedimento físico o mental, torpeza, menosprecio por el peligro, resentimiento, desatención a la instrucción, indiferencia, exceso de confianza, distracción, prisa, impaciencia, juego, fatiga y tedio" (EL ESPECTADOR, 1973. p. 2).

\section{La producción y el trabajo}

asalariado se constituyeron

como uno de los escenarios en

los cuales se instaló la

tecnología del cuidado de sí, en

la medida que la fuerza de

trabajo que representaban los varones adultos jóvenes, debía controlarse, amoldarse a las

necesidades del sistema económico.
Las causas descritas se relacionan con algunas características hegemónicas de la masculinidad en el país, por lo que es plausible entender por qué este poder sobre la vida - expresado en una actitud instituida como posibilidad de desarrollar prácticas de cuidado en el ámbito laboral - se expresa en el interés por dominar el cuerpo masculino para que este sea dócil, manejable. Es una mirada centrada en lo que Foucault denominó el cuerpo como máquina, moldeado mediante la educación, "el desarrollo de sus aptitudes, el arrancamiento de sus fuerzas, el crecimiento paralelo de su utilidad y su docilidad, su integración en sistemas de control eficaces y económicos" que garantizaran su productividad, su aporte al crecimiento del capital. Esto es, la "anatomopolítica del cuerpo humano" (FOUCAULT, 2007, p. 231). Pero el discurso de la seguridad industrial se profesionaliza en 1983, desarrollándose la carrera universitaria y se transforma en un campo de conocimiento denominado Salud Ocupacional, normalizado mediante el Decreto 614 del 14 de marzo de 1984, con el fin de "sentar las bases para la creación de un plan nacional unificado en el campo de la prevención de accidentes y enfermedades del trabajo, y el mejoramiento de las condiciones en el mismo" (EL ESPECTADOR, 1985. p. 12A-13A).

De acuerdo con lo anterior, el cuidado de sí en la salud adquiere forma y se construye en el ámbito de la produccion mediante cinco estrategias "de seguridad y/o de control", sustentadas en la teoría de los riesgos para la salud, en la que se concibe este "como efecto, probabilidad de ocurrencia de una patología en una población determinada, expresado a través del indicador paradigmático de incidencia" (DE ALMEIDA FILHO, 2009, p. 323-344). Esto es, como probabilidad de que se produzca un resultado adverso, o como factor que aumenta esa probabilidad. De acuerdo con esta teoría, las estrategias a las que se alude son: la prevención, que busca mantener informados y advertidos a los trabajadores sobre los riesgos y peligros que pueden presentarse e instruirlos para asumir actitudes y procedimientos a seguir en determinados momentos; la disuasión, que busca inducirlos a que desistan de determinadas conductas de riesgo que pueden vulnerarlos, busca efectos psicológicos; la vigilancia, que mediante el empleo de los sentidos humanos, busca detectar cualquier peligro y alertar para que se de respuesta exitosa ante el riesgo; la protección, entendida como aquellos elementos humanos, materiales o técnicos, que se emplean para defender, auxiliar, resistir, rechazar o reprimir cualquier ataque contra la persona; por último el control, como mecanismo verificador del uso adecuado de las normas y sistemas de vigilancia y protección, así como para evitar o detectar un riesgo o peligro (ARBELÁEZ, 1987).

El cuerpo masculino se protege y se mantiene mediante programas de salud ocupacional, para garantizar buenos niveles de productividad. Mediante estrategias de prevención, se estructura toda una complejidad de acciones, tales como el desarrollo de actividades deportivas, recreativas, el examen permanente del cuerpo, la garantía de la seguridad social para los trabajadores, se busca potenciar la capacidad productiva de los hombres. 
Adicionalmente, se desarrollan programas de seguridad industrial orientados a identificar el panorama de riesgos, accidentes y enfermedades profesionales con el fin de reducirlas o eliminarlas. Este está compuesto por tres áreas fundamentales: seguridad industrial, higiene industrial y medicina del trabajo, que conjuntamente permiten el desarrollo de las técnicas de prevención, disuasión, vigilancia, protección y control en las empresas y ámbitos de producción en general (EL ESPECTADOR, 1983). La seguridad y la protección contra el riesgo en el trabajo se convierte en un discurso racionalizador que busca, mediante una tecnología del cuidado de sí el control, no solo del espacio laboral del trabajador y de su movilidad en él, sino también de la vida familiar y social de éste, así como su propia regulación. Es decir, la racionalidad del discurso que circula en el trabajo, en relación con el cuidado de la salud durante la segunda mitad del siglo 20 , se convierte en un modelo regulador que ha sufrido transformaciones dirigidas a racionalizar todos los aspectos de la vida social, organizando deliberadamente los sujetos para garantizar su funcionamiento. La concentración social dentro de un marco nacional se convierte en un denso nexo de aparatos donde las instituciones de control social se volvían interconectadas. La autorregulación se convirtió en una alternativa democrática a la jerarquía y la coerción externa (FRASER, 2003), lo que ha hecho más efectivo el mecanismo de control, en la medida en que se induce, se instala subrepticiamente en las mentes y se legitima, se naturaliza como pauta que conduce la vida: "En efecto, al someter los individuos como agentes de control social, aunque al mismo tiempo se promueve su autonomía, se busca reemplazar la coerción externa con autorregulación interna" (FRASER, 2003. p. 33). Pero, durante las décadas de los 1980 y los 1990, se complejizan estos mecanismos, debido a que la globalización los transforma, generando estrategias de regulación social más privatizadas y dispersas que el anterior, en razón del sistema económico neoliberal que devino con nuevas exigencias de control y dominio, asociado a la necesidad de masificar las estrategias, es decir, se transita entre el disciplinamiento de los cuerpos para el trabajo y el control de la población trabajadora, en la medida que se considera "la forma, la naturaleza, la extensión, la duración, la intensidad de las enfermedades" que aquejan la población económicamente activa y se las trata como factores de riesgo permanentes que sustraen fuerzas, disminuyen los tiempos para el trabajo, minimizan las energías, conllevan costos económicos elevados, tanto por lo que deja de producirse como por los cuidados que pueden requerir. En suma, "la enfermedad como fenómeno de población, ya no como la muerte que se abate brutalmente sobre la vida - la epidemia - sino como la muerte permanente, que se desliza en la vida, la carcome constantemente, la disminuye y la debilita" (FOUCAULT, 2001, p. 217).

La lógica económica se otorga entonces la potestad de controlar la vida de la PEA, al instalar mecanismos de regulación cotidiana, con el apoyo de la ciencia y de disciplinas científicas, decide quienes deben vivir y quienes deben morir. Se instala una lógica perversa que busca asegurar la población para el trabajo con base en un análisis economicista de actividades que no son económicas y que constituyen las formas de vida de los seres humanos.

\section{Mecanismos de efectos periféricos}

En este conjunto de mecanismos es necesario tener claridad sobre dos aspectos que condicionan su alcance real en la población masculina. En primer lugar, el limitante que representa la focalización de las políticas públicas en Colombia, incluida la salud, por lo que las acciones en este ámbito se direccionan preferiblemente a sectores poblacionales vulnerables, tales como mujeres, menores y tercera edad. En segundo lugar, se perpetúa la posición tradicional entre hombres y mujeres según el género, asociada a los roles de producción y reproducción respectivamente, en el diseño y la implementación de las acciones e intervenciones desde las políticas públicas. Dado lo anterior, las estrategias de operación de estos mecanismos se pueden subdividir en dos grupos: las dirigidas al mantenimiento del cuerpo y las referidas a las restricciones de la sexualidad y la reproducción.

\section{Estrategias de mantenimiento del cuerpo}

En este conjunto de estrategias, se hace alusión en primer lugar a la higiene del cuerpo (PEDRAZA, 2011), como lineamiento central desde finales del siglo 19 y hasta los setenta incluso, en los discursos de la salud pública, a través de la Junta Central de Higiene como organismo estatal encargado del tema de la salud en el país (QUEVEDO, 1990). Se instaura un modelo higienista en la salud pública que busca, en relación con el cuidado de la salud, desarrollar prácticas preventivas contra enfermedades infectocontagiosas tales como el pián, la tuberculosis, la malaria, la fiebre amarilla, entre otras. Con respecto a la salud y la higiene, se señala que la salud es un capital invaluable y la economía de los individuos, las familias y los pueblos, está íntimamente en relación con ella. Por sus posibilidades de producción y consumo, que son consecuencia del bienestar en gran manera, cada vida adquiere 
un valor más elevado, mientras más progreso y cultura haya en la colectividad tiene también estrecha relación con la economía. La higiene en todo el mundo está vinculada a la agricultura y en nuestro medio solo en algunos sectores. Las posibilidades de la última son determinadas por la primera. Las demás industrias tienen también importantes relaciones. Es pues, base cierta del bienestar colectivo (EL COLOMBIANO, 1953).

En segundo lugar, la alimentación y nutrición cobran importancia para el mantenimiento del cuerpo y su utilidad para el trabajo. En esta medida se especifican acciones dirigidas a la cualificación del estado nutricional de la población colombiana, aduciéndose que la desnutrición afecta la capacidad y la productividad en el trabajo de las personas. Se hace énfasis en la importancia del mejoramiento de la dieta para elevar la productividad del trabajador y la vida promedio de la población (COLOMBIA, 1974).

La intencionalidad e interés por erradicar la malnutrición y por cambiar los hábitos alimenticios de los y las colombianas, estaba dado por los costos que podría implicar para la economía del país (ESCOBAR, 2007), el debilitamiento de los cuerpos. Así, bajo una lógica costo-beneficio, se afirma que el problema de la desnutrición que aqueja al pueblo colombiano, no solo disminuye su bienestar biológico, obstaculizando la elevación de los índices educativos, técnicos y de productividad que son base de su capacidad para generar ingreso, sino que disminuye la eficacia de los esfuerzos que el Estado realiza en campos tan variados como la salud, la educación y el desarrollo agrícola (COLOMBIA, 1974). En vista de la necesidad de ejercer control sobre la población más afectada por la malnutrición, sobre todo niños y niñas, madres y personas de la tercera edad, se instaura desde los setenta y se mantiene hasta los noventa incluso, el Plan Nacional de Alimentación y Nutrición (PAN), desde el que se desarrollan acciones dirigidas no solo a la población infantil y a las madres, sino también a la población económicamente activa, sustentando estas acciones en el hecho de que la solución del problema de la desnutrición afectará positivamente la capacidad productiva no solo de la actual generación sino de las generaciones futuras (COLOMBIA, 1974). La alimentación y la refinación alimentaria pasa así también por el rasero de la racionalidad económica, en tanto la relación del niño con el alimento representa una inversión mensurable en el tiempo que se revierte en la renta que este producirá una vez ingrese a la fuerza laboral, esta es la ganancia potencial del capital invertido en su alimentación (FOUCAULT, 2008).

En tercer lugar, se desarrollan acciones estatales dirigidas a atender el consumo de alcohol, sustancias psicoactivas, tabaco, entre otros. Pero, la no especificación del género resta alcance a las políticas y la hace difusa porque desdibuja su intencionalidad para sectores poblacionales masculinos. Se debiera partir del reconocimiento de que estos consumos son prácticas realizadas principalmente por los varones, por tanto la política debería albergar acciones específicas para estos. Solo se reconoce que una educación incompleta y divorciada de las necesidades más urgentes de la vida cotidiana disminuye en la población de bajos ingresos la posibilidad de prevenir diversos tipos de enfermedades. Hábitos alimentarios inadecuados se relacionan con la desnutrición. El alcoholismo y la drogadicción han alcanzado niveles críticos en jóvenes y adultos, y no son ajenos a enfermedades y muertes producidas por accidentes y homicidios (COLOMBIA, 1982).

Adicionalmente, se da prelación a los sectores poblacionales considerados vulnerables en la implementación de la política. En este sentido, es necesario considerar las tendencias focalizadoras de estas, lo que hace menos probable que los varones sean incluidos en ellas, por no considerarse como grupo vulnerable, aunque si se hace énfasis en atender la población económicamente activa. En este sentido, en el estudio no se incluyen categorías como la etnia o la clase social, cuya reflexión implicaría variaciones en la reflexión que se propone. Se apuntalan entonces para la intervención estrategias como la prevención de las enfermedades, el aumento de la cobertura de los servicios asistenciales a los grupos más desfavorecidos y la mejora de su calidad son, de una parte, objetivos que tienden a lograr mayor equidad social y, de otra, objetivos estratégicos para elevar la productividad en el trabajo, lo cual depende en buena medida de la salud de la población (COLOMBIA, 1982).

En esta misma línea, a partir de los noventas se estructuran acciones desde lo que se llamó el Plan Nacional de Atención Primaria, referidas a la promoción de la salud y prevención de la enfermedad que incluyen campañas educativas relacionadas con el cambio de hábitos, haciendo énfasis en las prácticas de autocuidado de la salud.

\section{Mecanismos de restricciones a la sexualidad}

En los años 1980 se evidencia una creciente preocupación en el ámbito mundial por la aparición del Síndrome de Inmunodeficiencia Adquirida SIDA y, en el año de 1986, se impulsa desde el Ministerio de Salud "un proyecto de prevención y control de esa enfermedad, que se orienta a detectar precozmente los portadores y enfermos, para interrumpir la cadena de transmisión, mediante procedimientos de control en bancos de sangre y la producción de derivados sanguíneos" (COLOMBIA, 1986, p. 44). Pero es a partir de los años 1990, con la reforma constitucional de 1991, que se desarrollan con mayor solidez programas y campañas de prevención y educación sobre el 
SIDA. Se privilegia para ello la población joven escolarizada y consistió en una iniciativa que involucró la formación de núcleos de adolescentes que estuvieran en disposición de difundir información en torno a la manera de prevenir el contagio de la enfermedad (EL ESPECTADOR, 1996). Con la epidemia del VIH-SIDA, surge la necesidad de regular la sexualidad desde el Estado colombiano, por ello en el año de 1993 se diseña un plan aprobado por el Consejo de Política Económica y Social (CONPES), que incluye programas de televisión, distribución gratuita de condones, aportes presupuestales elevados, nueva legislación y cátedras escolares y universitarias sobre educación sexual, para "luchar contra la epidemia del SIDA" (HERRERA, 1993. p. 10).

Si bien los mecanismos de restricciones a la sexualidad involucran tanto a hombres como a mujeres, no es clara la perspectiva de género necesaria para el desarrollo de estos procedimientos, considerando los modelos hegemónicos de masculinidad en el país, que a muchos de los varones les impide una actitud proactiva en relación con el cuidado de su salud. Este hecho y la constante de las directrices de las políticas hasta el año 2000, sustentadas en la focalización y prelación de grupos vulnerables, restan alcances mayores en la población varonil. Debe insistirse en el hecho de que entre los hombres se encuentran las tasas más elevadas de infección por VIH-SIDA en el país, pero desde las políticas se les ubica como responsables del malestar de las mujeres, en lugar de potenciar su reflexión en torno a sus responsabilidades y derechos como sujetos generizados.

\section{Reflexiones Finales}

Desde el Trabajo Social, con la finalidad de dar mayor solidez y claridad a sus intervenciones en el escenario de la salud pública en la contemporaneidad, debe comprenderse como las estrategias y tácticas del cuidado de sí en la salud han sido delimitadas por discursos, desde hace ya más de medio siglo, en los que se apuntala la idea de progreso como un elemento necesario para mejorar la competitividad del país en el exterior. Tal idea ha conllevado grandes exigencias al Estado Colombiano, sustentadas en la instauración de la modernización de la economía para la consabida implantación del modelo capitalista en nuestro territorio, por lo que se privilegió el crecimiento como punto de partida para la toma de decisiones en el ámbito político.

Durante las cinco décadas se presenta entonces una constante en los discursos del desarrollo en el país, referida al hecho de que en este periodo se observa en los planes nacionales, una orientación hacia la necesaria articulación entre las políticas sectoriales y el espíritu general de éstos, apuntalada en el crecimiento de la economía. Este tipo de discursos se sustenta en teorías tales como los estados de crecimiento económico, hegemónicas hasta casi la mitad de la década de los 1970 cuando incursionan las teorías del capital humano como eje articulador de las decisiones nacionales, en lo relacionado con las políticas públicas y, por supuesto, con la salud. Estos hechos posibilitan al Trabajo Social en salud pública comprender las lógicas de funcionamiento interno de las acciones en salud y sus repercusiones en el bienestar de los colectivos desde una perspectiva estructural, que condiciona comportamientos, conductas y aprendizajes basados en el género. Lo anterior condiciona la salud a las lógicas económicas al introducir en este ámbito un discurso racionalizador del costo-beneficio al ver ésta como una inversión y no como un gasto. El binomio economía-salud cobra fuerza en estas décadas, hecho que supedita las decisiones en salud a los requerimientos de la economía, pues se considera que una buena salud, procura mejores condiciones para la población económicamente activa - varones adultos jóvenes en su mayoría - y, por tanto, posibilita el aumento de la productividad nacional. Opera así una racionalidad que ha conllevado la gestación del cuidado de sí en la salud masculina como tecnología de poder que, durante la segunda mitad del siglo 20, se articula principalmente a la productividad y el crecimiento económico, reafirmando la división sexual del trabajo, que instala a los varones en el ámbito de la producción y a la mujer en el ámbito doméstico.

Desde esta lógica se teje la red de poder en relación con el objeto de estudio propuesto. Se instaura un poder-saber que controla y regula los cuerpos masculinos, que los obliga a encauzarse alrededor de los intereses del capital y de la productividad. Se desdibuja el privilegio y se visibiliza la vulnerabilidad de los hombres en el cumplimiento de un rol asignado socioculturalmente de Trabajador-Proveedor. La producción y el trabajo asalariado se constituyeron como uno de los escenarios en los cuales se instaló la tecnología del cuidado de sí, en la medida que la fuerza de trabajo que representaban los varones adultos jóvenes, debía controlarse, amoldarse a las necesidades del sistema económico.

Los anteriores planteamientos se ubican como ejes de análisis que aportan a la comprensión del contexto socio-político y económico que ha regido a Colombia, no solo durante la segunda mitad del siglo 20, sino aún en la actualidad, para el diseño e implementación de políticas públicas orientadas al cuidado de sí en la salud masculina, los cuales se constituyen como condicionantes para el desempeño de los y las Trabajadoras Sociales y de otras áreas afines para el desarrollo de acciones de educación, prevención y promoción, y deben ser considerados en el análisis de la realidad en salud hoy y su consecuente problematización para el desempeño desde nuestra profesión. 


\section{Referencias}

ARBELÁEZ, H. Técnicas de seguridad. Períodico El Espectador, Bogotá, p. 8D, junio 1987.

COLOMBIA. Plan de desarrollo 1974-1978: para cerrar la brecha. Disponible en: https://www.dnp.gov.co/PND/ PlanesdeDesarrolloanteriores.aspx. Acceso en: 22 feb. 2009.

Plan de desarrollo 1982-1986: cambio con equidad. Disponible en: https://www.dnp.gov.co/PND/PlanesdeDesarrollo anteriores.aspx. Acceso en: 22 feb. 2009.

Plan de desarrollo 1986-1990: Plan de economía social. Disponible en: https://www.dnp.gov.co/PND/PlanesdeDesarrollo anteriores.aspx. Acceso en: 22 feb. 2009.

. Plan de desarrollo 1990-1994: la revolución pacífica. Disponible en: https://www.dnp.gov.co/PND/PlanesdeDesarrollo anteriores.aspx. Acceso en: 22 feb. 2009.

Plan de desarrollo 1994-1998: el Salto Social. Disponible en: https://www.dnp.gov.co/PND/PlanesdeDesarrolloanteriores.aspx.

Acceso en: 22 feb. 2009.

CUBIDES CIPAGAUTA, H. Foucault y el sujeto político. Ética y el cuidado de sí. Bogotá D.C.: Siglo del Hombre Editores, 2006.

DE ALMEIDA FILHO, N.; AYR, J. R. Riesgo: concepto básico de la epidemiología. Revista Salud colectiva. Lanús, v. 3, n. 3, p. 323-

344, Septiembre/Diciembre 2009.

EL COLOMBIANO. En la higiene del hogar se encuentra la salud. Períodico El Colombiano, Bogotá, p. 16, 4 ago. 1951. En toda forma el baño es provechoso. Períodico El Colombiano, Bogotá, p. 4, 6 ago. 1951.

. Higiene del Vestido. Períodico El Colombiano, Bogotá, p. 4, 14 jun. 1954.

. La salud protegida por la higiene. Períodico El Colombiano, Bogotá, p. 15, 20 jul. 1953.

EL ESPECTADOR. Baja considerable en los índices de accidentalidad. Periódico El Espectador, Bogotá, p. 2D, 22 jun. 1970. . Educación contra el VIH. Períodico El Espectador. Bogotá, p. 2D, 11 jul. 1996.

. Educación contra el VIH. Períodioco El Espectador. Bogotá, p. 2D, 11 jun. 1996.

. El accidente es una forma de enfermedad. Periodico El Espectador, Bogotá, p. 5, 9 oct. 1961.

. En marcha la salud ocupacional. Períodico El Espectador, Bogotá, p. 5C 24 jun. 1987.

. Limpieza, una de las bases de seguridad. Periódico El Espectador, Bogotá, p. 6D, 1 sep. 1971.

. Los equipos adecuados de protección personal. Periódico El Espectador, Bogotá, p. 2, 10 jul. 1973.

Los peligros de los metales. Para su taller tenga en cuenta. Periódico El Espectador, Bogotá, p. 6, 10 mar. 1960.

Se profesionaliza la seguridad industrial. Períodico El Espectador, Bogotá, p. 12A-13A, 20 feb. 1985.

ESCOBAR, A. La invención del tercer mundo. Construcción y deconstrucción del desarrollo. Caracas: Fundación Editorial el perro y la rana, 2007.

FLÓREZ, N. Las transformaciones sociodemográficas en Colombia durante el siglo XX. Santafé de Bogotá: Tercer Mundo EditoresBanco de la República, 2000.

FOUCAULT, M. Defender la sociedad. Buenos Aires: Fondo de Cultura Económica de Argentina; 2001.

. Historia de la sexualidad. La voluntad de saber. México D.F.: Siglo XXI Editores S.A.; 2007.

. Nacimiento de la biopolítica. Buenos Aires: Fondo de la Cultura Económica Argentina; 2008.

FRASER, N. ¿De la disciplina hacia la flexibilización? Releyendo a Foucault bajo la sombra de la globalización. Revista Mexicana de Ciencias Políticas y Sociales. México, v. XLVI, n. 187, p. 15-33, 2003.

HERRERA, R. Revelan el Plan anti-sida. En la lucha participan Gobierno, ONGs y Comunidad. Períodico El Espectador. Bogotá, p. 10A, 24 jul. 1993.

LÓPEZ, A. F. Lauchin Currie y el desarrollo colombiano. Revista Criterio Libre. Bogotá Facultad Ciencias Económicas Universidad Libre, v. 9, n. 14, p. 21-42, Enero/Junio 2011.

PEDRAZA, G. Z. En cuerpo y alma, visiones del progreso y de la felicidad. Educación, cuerpo y orden social en Colombia (1830-1990). Bogotá: Ediciones Uniandes, 2011.

Peláez M M, Rodas L S. La política de género en el Estado Colombiano: Un camino de conquistas sociales. Medellín: Centro Interdisciplinario de Estudios de Género -CIEG-, Universidad de Antioquia; 2002. p. 3-353.

QUEVEDO, V. Análisis sociohistórico. En YEPES, F. J. La salud en Colombia. Estudio Sectorial de salud. Documento general. Tomo I. Bogotá D.E.: Ministerio de Salud. Departamento Nacional de Planeación, p. 111-274, 1990.

\section{Nota}

1 Este trabajo es parte de la tesis doctoral en Salud Pública titulada: Varones y Cuidado de sí: los costos del privilegio para la salud masculina. Universidad de Antioquia, Facultad Nacional de Salud Pública Hector Abad Gómez, Medellín-Colombia, Mayo de 2013. 


\section{Nora Eugenia Muñoz Franco}

noramunozfranco@yahoo.es

Doctora em Salud Pública

Professora do Departamento de Serviço Social, Faculdade de Ciências Sociais e Humanas da Universidade de Antioquia.

\section{Universidade de Antioquia}

Ciudad Universitaria

Calle 67, n. 53-108, Bloque 9, Oficina 226

Medellín-Colombia 